\title{
Xyloketal B exerts antihypertensive effect in renovascular hypertensive rats via the NO-sGC-cGMP pathway and calcium signaling
}

\author{
Li-yan ZHAO ${ }^{1, \#}$, Jie LI, \#, Xiong-qing HUANG, "\#, Guo-hao WANG ${ }^{4}$, Xiao-fei $\mathrm{LV}^{5}$, Wei-feng MENG ${ }^{5}$, Wen-liang $\mathrm{CHEN}^{6}$, \\ Ji-yan PANG ${ }^{7}$, Yong-cheng LIN $^{7}$, Hong-shuo SUN ${ }^{8}$, Guan-lei WANG ${ }^{5,9, *}$, Yao-min DU ${ }^{10, *}$ \\ ${ }^{1}$ Department of Pharmacy, The First Affiliated Hospital of Sun Yat-sen University, Guangzhou 510080, China; ${ }^{2}$ Department of \\ Anesthesiology, The Second Affiliated Hospital of Guangzhou University of Chinese Medicine, Guangzhou 510120, China; ${ }^{3}$ Department \\ of Anesthesiology, The First Affiliated Hospital, Sun Yat-sen University, Guangzhou, China; ${ }^{4}$ Department of Computer Engineering, \\ Engineering Institute in Lishui University, Lishui 323000, China; ${ }^{5}$ Department of Pharmacology, Zhongshan School of Medicine, Sun \\ Yat-sen University, Guangzhou 510080, China; ${ }^{6}$ Department of Pharmacology, School of Pharmaceutical Sciences, and the Fifth \\ Affiliated Hospital, Guangzhou Medical University, Guangzhou 511436, China; ${ }^{7}$ Department of Applied Chemistry, School of Chemistry \\ and Chemical Engineering, Sun Yat-sen University, Guangzhou 510080, China; ${ }^{8}$ Departments of Surgery and Physiology, Institute of \\ Medical Science, Faculty of Medicine, University of Toronto, Toronto, ON M5G 1G6, Canada; ${ }^{9}$ Guangdong Province Key Laboratory of \\ Functional Molecules in Oceanic Microorganism (Sun Yat-sen University), Bureau of Education, Guangzhou, China; ${ }^{10}$ Departments of \\ Geriatrics, Guangdong Academy of Medical Sciences, Guangdong General Hospital, Guangzhou 510080, China
}

\begin{abstract}
Xyloketal B (Xyl-B) is a novel marine compound isolated from mangrove fungus Xylaria sp. (№ 2508). We previously showed that Xyl-B promoted endothelial NO release and protected against atherosclerosis through the Akt/eNOS pathway. Vascular NO production regulates vasoconstriction in central and peripheral arteries and plays an important role in blood pressure control. In this study, we examined whether Xyl-B exerted an antihypertensive effect in a hypertensive rat model, and further explored the possible mechanisms underlying its antihypertensive action. Administration of Xyl-B (20 mg. $\mathrm{kg}^{-1} \cdot \mathrm{d}^{-1}$, ip, for 12 weeks) significantly decreased the systolic and diastolic blood pressure in a two-kidney, two-clip (2K2C) renovascular hypertensive rats. In endothelium-intact and endotheliumdenuded thoracic aortic rings, pretreatment with Xyl-B (20 $\mu \mathrm{mol} / \mathrm{L})$ significantly suppressed phenylephrine (Phe)-induced contractions, suggesting that its vasorelaxant effect was attributed to both endothelial-dependent and endothelial-independent mechanisms. We used SNP, methylene blue (MB, guanylate cyclase inhibitor) and indomethacin (IMC, cyclooxygenase inhibitor) to examine which endothelial pathway was involved, and found that MB, but not IMC, reversed the inhibitory effects of Xyl-B on Phe-induced vasocontraction. Moreover, Xyl-B increased the endothelial NO bioactivity and smooth muscle cGMP level, revealing that the NO-sGCcGMP pathway, rather than $\mathrm{PGl}_{2}$, mediated the anti-hypertensive effect of Xyl-B. We further showed that Xyl-B significantly attenuated $\mathrm{KCl}$-induced $\mathrm{Ca}^{2+}$ entry in smooth muscle cells in vitro, which was supposed to be mediated by voltage-dependent $\mathrm{Ca}^{2+}$ channels (VDCCs), and reduced ryanodine-induced aortic contractions, which may be associated with store-operated $\mathrm{Ca}^{2+}$ entry (SOCE). Taken together, these findings demonstrate that Xyl-B exerts significant antihypertensive effects not only through the endothelial NO-sGCcGMP pathway but also through smooth muscle calcium signaling, including VDCCs and SOCE.
\end{abstract}

Keywords: hypertension; two-kidney, two-clip renovascular hypertensive rats; aortic rings; smooth muscle cells; xyloketal B; NO-sGCcGMP pathway; SNP, methylene blue; indomethacin; KCl; ryanodine

Acta Pharmacologica Sinica (2018) 39: 875-884; doi: 10.1038/aps.2018.12; published online 29 Mar 2018

\footnotetext{
\#These authors contributed equally to this work.

*To whom correspondence should be addressed.

E-mail duymgams@163.com (Yao-min DU); wangglei@mail.sysu.edu.cn (Guan-lei WANG).

Received 2017-11-04 Accepted 2018-01-12
}

\section{Introduction}

Hypertension is a major risk factor that predisposes patients to cardiovascular disorders and has high rates of morbidity and mortality. Emerging data implicate increases in systemic oxidative stress $^{[1]}$ and vascular inflammation ${ }^{[2]}$ in the pathogene- 
sis of hypertension. Endothelial dysfunction, which manifests as impaired nitric oxide (NO) bioactivity, is an important early event associated with impaired vessel diastolic function ${ }^{[3]}$ and mainly results from increased nitric oxide $(\mathrm{NO})$ degradation due to interactions between $\mathrm{NO}$ and superoxide anions. Pharmacological approaches to restore endothelial function or complement NO have been demonstrated to have antihypertensive effects.

Marine organisms are abundant natural sources of novel bioactive compounds because they can produce a variety of molecules with unique structural features and exhibit various types of biological activities. Xyloketal B (Xyl-B), obtained from the South China Sea Coast, is one of a series of novel ketal compounds isolated from the mangrove fungus Xylaria $s p\left(\mathrm{No}_{\mathrm{o}} 2508\right)^{[4,5]}$. In previous studies, multiple biomedical activities of Xyl-B have been demonstrated, including neuroprotective effects against toxicity ${ }^{[6]}$, antioxidant effects on endothelial cells ${ }^{[7]}$ and zebrafish ${ }^{[8]}$, anti-glioma effects ${ }^{[9]}$ and reduced atherosclerosis plaque formation in apolipoprotein E-deficient mice ${ }^{[10]}$. After exploring the underlying mechanisms, we have found that Xyl-B can directly scavenge DPPH free radicals, promote endothelial $\mathrm{NO}$ releas $\mathrm{e}^{[7]}$ and increase eNOS phosphorylation at Ser-1177 in a concentration- and time-dependent manner ${ }^{[10]}$. Furthermore, the effect of Xyl-B on aortic tension was also evaluated, and the data indicated that Xyl-B can improve NO-dependent aortic vasorelaxation in atherosclerotic $\mathrm{ApoE}^{-/-}$mice ${ }^{[10]}$ and exert potent vasorelaxant activity on $\mathrm{KCl}$-induced contractions in isolated rat aortic rings ${ }^{[11]}$. Based on these findings, we hypothesize that Xyl-B may regulate vascular tone through the endothelial NO system and thus has an effect on blood pressure control.

Therefore, in the present study, we established a 2-kidney, 2-clip $(2 \mathrm{~K} 2 \mathrm{C})$ renovascular hypertensive rat model to evaluate the effects of Xyl-B in the development of hypertension. In addition, we further explored the possible mechanisms for the antihypertensive effects of Xyl-B, including Xyl-B-mediated vasorelaxation, endothelial nitric oxide synthase (eNOS) bioactivity, the NO-sGC-cGMP pathway and the prostacyclin pathway. Abnormal calcium signaling has been demonstrated to regulate arterial tone in the development of hypertension in both human patients and animal models. Consequently, $\mathrm{Ca}^{2+}$ channel blockers are commonly used as anti-hypertensive agents. Because we found that Xyl-B suppressed $\mathrm{KCl}$ - and phenylephrine (Phe)-induced contractions in endotheliumdenuded thoracic aortic rings, which could not be completely explained by the endothelium-dependent pathway, the influence of Xyl-B on calcium signaling in vascular smooth muscle cells was also investigated.

\section{Materials and methods}

\section{Chemicals and reagents}

Xyl-B was isolated by the Department of Applied Chemistry, Sun Yat-sen University (Guangzhou, China). The identity and purity of the compound were characterized by HPLC and ${ }^{2} \mathrm{D}-\mathrm{NMR}$ as previously described ${ }^{[4]}$. Xyl-B was dissolved in dimethyl sulfoxide (DMSO) and stored at $-20^{\circ} \mathrm{C}$ until use.
The final concentration of DMSO in culture media was less than $0.1 \%$. Phenylephrine (Phe), acetylcholine (ACh), sodium nitroprusside (SNP) and $N^{\mathrm{G}}$-nitro-L-arginine methyl ester (L-NAME) were purchased from Sigma (St Louis, MO, USA). Methylene Blue was obtained from Merck (Massachusetts, USA). $\left[{ }^{3} \mathrm{H}\right]$ L-arginine was obtained from Beijing Institute of Nuclear Industry (Beijing, China). All other reagents used were purchased from Sigma unless otherwise specified.

\section{Animals and experimental design}

All experimental animal procedures and protocols were approved by the Sun Yat-sen University Committee for Animal Research and were conducted in accordance with the National Research Council's guidelines. 2K2C stroke-prone renovascular hypertension was induced in male SpragueDawley rats (60-80 g, purchased from the Experimental Animal Center of Sun Yat-sen University) as described previously $^{[12,13]}$. All rats were randomly divided into 5 groups: (1) the Sham group: sham-operated control group; (2) the Htn group: 2K2C-operated hypertensive group; (3) the propylene glycol-treated hypertensive $(\mathrm{H}+\mathrm{t}+\mathrm{Sol})$ group: the rats received an intraperitoneal injection of $2 \mathrm{~mL}$ of saline solution containing $20 \%$ propylene glycol once daily after surgery; (4) the Htn $+\mathrm{Xyl}-\mathrm{B}$ group: $2 \mathrm{~K} 2 \mathrm{C}$ rats received an intraperitoneal injection of Xyl-B (20 mg kg-1 $\mathrm{d}^{-1}$ in $2 \mathrm{~mL}$ of saline solution containing $20 \%$ propylene glycol) after surgery; and (5) the Htn+Cap group: $2 \mathrm{~K} 2 \mathrm{C}$ rats were treated with captopril $\left(0.1 \mathrm{mg} \mathrm{kg}^{-1} \cdot \mathrm{d}^{-1}\right.$, ig). The sham-operated animals underwent sham procedures without clip placement. Body weight and SBP or DBP measured by tail cuff plethysmography ${ }^{[13]}$ were recorded every two weeks until 12 weeks after operation.

\section{Vascular reactivity experiments in aortic rings}

Aortic rings were prepared and vascular reactivity experiments were performed as previously described ${ }^{[14]}$. Briefly, Sprague-Dawley rats (200-250 g) were euthanized and their aortas were quickly removed and placed into ice-cold Kreb's solution (composition in mmol/L: $\mathrm{NaCl} 119, \mathrm{KCl} 4.7, \mathrm{KH}_{2} \mathrm{PO}_{4}$ $1.18, \mathrm{MgSO}_{4} 1.17, \mathrm{NaHCO}_{3} 25, \mathrm{CaCl}_{2} 2.5$, EDTA 0.026, and glucose 5.5) where they were freed of fatty and connective tissues. Aortic ring segments (3-5 mm) were then cut and each segment was mounted onto separate Mulvany myograph chambers, which involved placing each ring onto two stainless hooks in an isometric myograph filled with Kreb's solution $(5 \mathrm{~mL})$ aerated with $95 \% \mathrm{O}_{2} / 5 \% \mathrm{CO}_{2}$ and maintained at $37^{\circ} \mathrm{C}$. Changes in isometric force were recorded online using a Multi-myograph data acquisition system with PowerLab software (AD Instruments, Inc). The rings were stretched to an optimal resting tension $(1.5 \mathrm{~g})$ and allowed to stabilize for $2 \mathrm{~h}$. An additional equilibration period of $30 \mathrm{~min}$ was allotted before treatment with any drugs. The rings were stimulated with Phe $(1 \mu \mathrm{mol} / \mathrm{L})$ when indicated to induce contraction. To examine the possible mechanisms of the vaso-relaxant effects of Xyl-B, the NO synthase (NOS) inhibitor L-NAME $(100 \mu \mathrm{mol} / \mathrm{L})$, the soluble guanylate cyclase inhibitor methylene blue (MB, $10 \mu \mathrm{mol} / \mathrm{L})$ or the cyclooxygenase inhibitor 
indomethacin (IMC, $10 \mu \mathrm{mol} / \mathrm{L})$ was used. The endotheliumintact aortic rings were pre-incubated with these inhibitors for 30 min before Xyl-B was added.

eNOS activity detection in human umbilical vascular endothelial cells (HUVECs)

Primary culture of HUVECs was performed using collagenase digestion of the human umbilical cord as described previously $^{[7]}$. Three to six generations of identified HUVECs were used in this experiment. Cells were pre-incubated with 20 $\mu \mathrm{mol} / \mathrm{L}$ of Xyl-B for $0,15,30,45$, or 60 min or with $0,10,20$, 40 , or $80 \mu \mathrm{mol} / \mathrm{L}$ of Xyl-B separately for $30 \mathrm{~min}$, and eNOS activity was evaluated by determining the conversion ratio of $\left[{ }^{3} \mathrm{H}\right] \mathrm{L}$-arginine to $\left[{ }^{3} \mathrm{H}\right] \mathrm{L}$-citrulline at $15 \mathrm{~min}$ or $45 \mathrm{~min}$ with a liquid scintillation counter (Packard Inc, CT, USA) ${ }^{[15]}$.

\section{Measurement of cyclic GMP in primary cultured VSMCs}

Vascular smooth muscle cells (VSMCs) were isolated from adult rat thoracic aortas by the explants method as previously described $^{[16]}$. Passages 3 to 6 showing $>99 \%$ positive immunostaining against a-smooth muscle-actin (a-SMA) were used for the experiments, and cells at $80 \%$ confluence were arrested by serum-starvation for $24 \mathrm{~h}$. Cyclic GMP (cGMP) levels in VSMCs were determined using Parameter ${ }^{\mathrm{TM}} \mathrm{Cyclic}$ GMP Assay kits (R\&D Systems, Minnesota, USA) according to the manufacturer's instructions. The cells were divided into a VSMC mono-culture group and a VSMC-HUVEC co-culture group. Each group of cells was treated with DMSO, SNP (10 $\mu \mathrm{mol} / \mathrm{L})$, or Xyl-B (20 and $40 \mu \mathrm{mol} / \mathrm{L})$ and the incubation time was $30 \mathrm{~min}$. None of the drugs exhibited cytotoxicity at any dose used (evaluated by MTT assay, data not shown).

\section{Determination of single-cell intracellular $\mathrm{Ca}^{2+}$ concentration} $\left(\left[\mathrm{Ca}^{2+}\right]_{\mathrm{i}}\right)$ in VSMCs

Intracellular $\mathrm{Ca}^{2+}$ was detected by Fluo-3/AM staining as described previously ${ }^{[17]}$. Cells plated in $35-\mathrm{mm}$ petri dishes were loaded with Fluo-3/AM (10 $\mu \mathrm{mol} / \mathrm{L}$, Sigma) in Kreb's solution at $37{ }^{\circ} \mathrm{C}$ for $30 \mathrm{~min}$ and then exposed to $\mathrm{KCl}$ (60 $\mathrm{mmol} / \mathrm{L})$. Xyl-B was added $5 \mathrm{~min}$ prior to $\mathrm{KCl}$ stimulation. The dishes were washed twice with Kreb's solution to remove unhydrolyzed indicator before transfer to a chamber where the drugs were added. Cellular real-time fluorescence $\left(E_{x 488 \mathrm{~nm}}\right.$, $\left.E_{\mathrm{m} 522 \mathrm{~nm}}\right)$ was measured using a confocal microscope (FV500IX 81, OLYMPUS, Japan). A change in fluorescence was expressed as $F / F_{0}$, where $F$ represents the fluorescence intensity $(F)$ of each pixel in the original fluorescence image and $F_{0}$ is defined as the $F$ value at the beginning of the images when the cell was assumed to be in a resting state.

\section{Statistical analysis}

All values are expressed as the means \pm SEM. The data were analyzed by a two-tailed unpaired Student's $t$-test between 2 groups and by one-way ANOVA followed by the Bonferroni post hoc test for 3 or more groups. The analyses were performed using GraphPad Prism Software (GraphPad Software Inc, La Jolla, CA, USA). A P value $<0.05$ was considered statis- tically significant.

\section{Results}

Xyl-B decreases blood pressure in $2 \mathrm{~K} 2 \mathrm{C}$ hypertensive rats

First, we assessed the antihypertensive effects of Xyl-B using the $2 \mathrm{~K} 2 \mathrm{C}$ hypertensive rat model and blood pressures were measured 0, 2, 4, 6, 8, 10 and 12 weeks after the operation. As shown in Figure 1A and $1 \mathrm{~B}$, systolic blood pressure (SBP) and diastolic blood pressure (DBP) in the hypertension (Htn) group increased progressively at 2, 4, 6, 8, 10 and 12 weeks after the operation, which is consistent with our previous report $^{[18,19]}$. Xyl-B at $20 \mathrm{mg} \cdot \mathrm{kg}^{-1} \cdot \mathrm{d}^{-1}$ was administered to the 2K2C rats intraperitoneally; saline solution containing $20 \%$
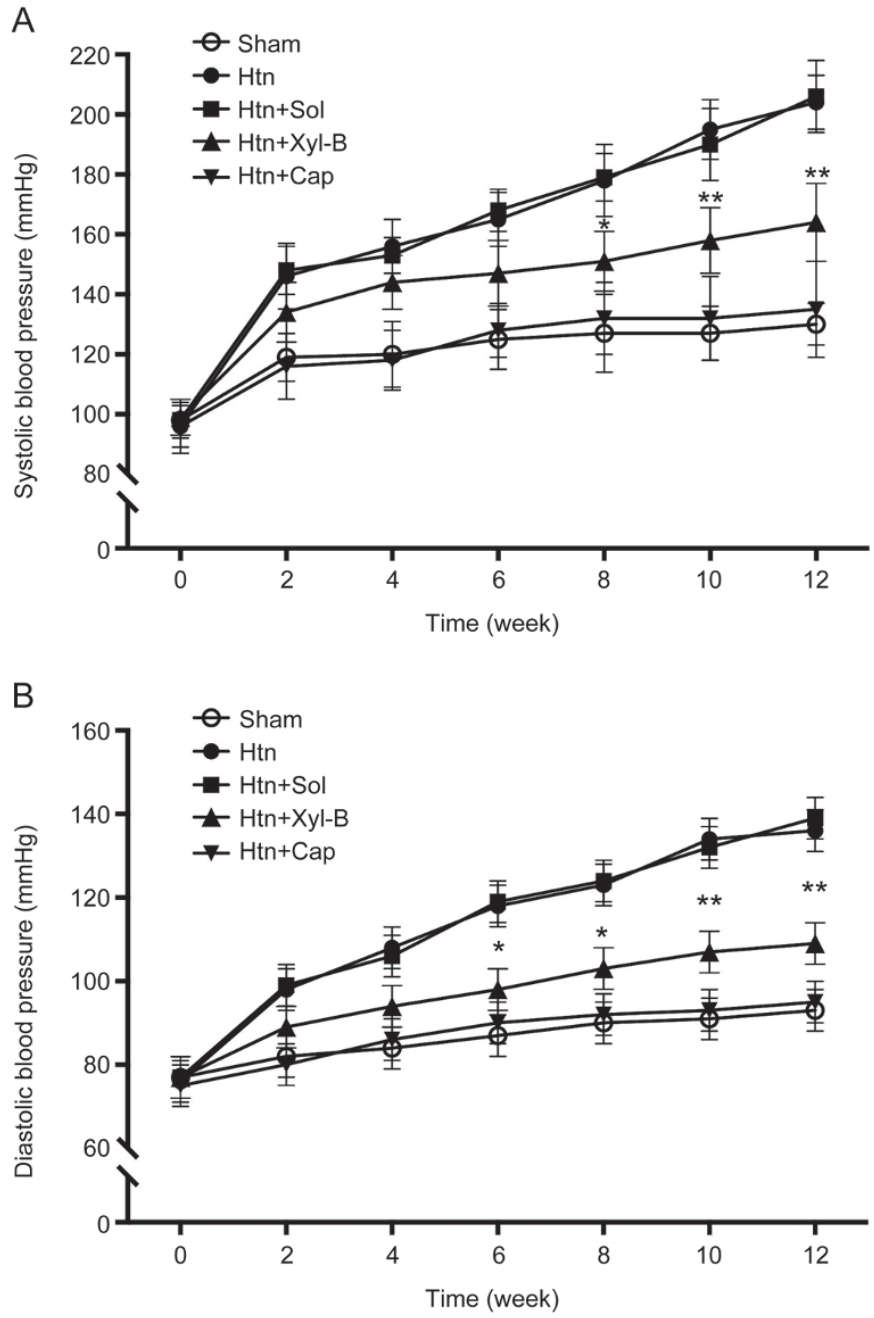

Figure 1. Effect of Xyl-B administration on blood pressure of 2-Kidney 2-Clip (2K2C) hypertensive rats. $2 \mathrm{~K} 2 \mathrm{C}$ hypertensive rat model was established and $20 \mathrm{mg} \cdot \mathrm{kg}^{-1} \cdot \mathrm{d}^{-1}$ of Xyl-B was administered intraperitoneally everyday while saline solution containing $20 \%$ propylene glycol and captopril were given as solvent and positive control respectively for 12 weeks. Systolic pressure (A) and diastolic pressure (B) were measured at 0 , 2, 4, 6, 8, 10 and 12 weeks after operation. Htn: 2K2C hypertension; Sol: saline solution containing $20 \%$ propylene glycol ( $2 \mathrm{~mL}$, ip); Cap: captopril (0.1 mg.kg $\cdot \mathrm{d}^{-1}$, ig). $n=12-14 .{ }^{*} P<0.05,{ }^{* *} P<0.01$ vs $\mathrm{Htn}+$ Sol group. 
propylene glycol $\left(2 \mathrm{~mL}\right.$, ip) and captopril $\left(0.1 \mathrm{mg} \cdot \mathrm{kg}^{-1} \cdot \mathrm{d}^{-1}\right.$, ig) were used as the solvent and the positive control, respectively. The solvent control $(\mathrm{Htn}+\mathrm{Sol})$ group maintained high SBP and DBP values, showing no significant differences from the age-matched Htn group $(n=12-14, P>0.05)$. Xyl-B significantly reduced the SBP and DBP of the $2 \mathrm{~K} 2 \mathrm{C}$ hypertensive rats at 8,10 and 12 weeks, with decreases of $59.57 \% / 65.63 \%$, $55.17 \% / 64.10 \%, 59.15 \% / 68.18 \%$ relative to the corresponding values in the positive control $(\mathrm{Htn}+\mathrm{Cap})$ group (SBP/DBP, $n=12-14, P<0.05$ vs the Htn group).

\section{Xyl-B reduces aortic tension in response to phenylephrine}

Xyloketals have been demonstrated to exert vaso-relaxant activities in $\mathrm{KCl}$-induced vasoconstriction that were stronger in endothelium-intact rings than in denuded rings, suggesting that the vaso-relaxant effect of these compounds may be mediated by endothelium-dependent mechanisms ${ }^{[11]}$. Hypertension is closely related to vascular tone, and our previous studies have suggested that Xyl-B plays an important role in endothelial NO synthesis, which mediates vessel relaxation. To further confirm whether Xyl-B protects against hypertension by affecting vascular tension, thoracic aorta constriction in response to Phe was measured. As shown in Figure 2, Xyl-B significantly inhibited Phe-induced vasoconstriction at concentrations of $10^{-6} \mathrm{~mol} / \mathrm{L}$ and $10^{-5} \mathrm{~mol} / \mathrm{L}$. Interestingly, the inhibition effect was partly reversed by L-NAME, the inhibitor of endothelial nitric oxide synthetases (eNOS); however, a significant difference was observed in vessel tension between the $X y l-B+L-N A M E$ group and the solvent control group at $10^{-5}$ $\mathrm{mol} / \mathrm{L}$.

\section{Xyl-B inhibits aortic vasoconstriction through the NO-sGC-cGMP pathway}

To identify the pathway through which Xyl-B exerts its antihypertensive effect, we first detected Xyl-B's influence on acetylcholine (ACh)- and sodium nitroprusside (SNP, an exogenous NO provider)-induced vessel diastole in Phe precontracted vessels. As shown in Figure 3, Xyl-B enhanced the vaso-relaxant effect of $\mathrm{ACh}$, but not $\mathrm{SNP}$, indicating that Xyl-B decreases vessel tension through the endothelium-dependent NO-related pathway, which is consistent with Figure 2.

Endothelium-dependent vasodilation is regulated primarily by $\mathrm{NO}$ but also by prostacyclin $\left(\mathrm{PGI}_{2}\right)$ and an unidentified endothelium-derived hyperpolarizing factor. $\mathrm{NO}$ and $\mathrm{PGI}_{2}$ are the predominant endogenous endothelial vaso-relaxant substances ${ }^{[20]}$. The NO-sGC-cGMP signaling cascade plays an essential role in vascular smooth muscle relaxation, and clinical studies indicate that endothelium-derived $\mathrm{NO}$ is involved in normal and pathological blood pressure regulation. $\mathrm{PGI}_{2}$ is produced by the cyclooxygenases, COX-1 and COX-2, which form the prostaglandin endoperoxide $\mathrm{PGG}_{2}$. This is converted to $\mathrm{PGH}_{2}$, which is then transformed enzymatically into $\mathrm{PGI}_{2}$ by prostacyclin synthase. To determine whether the $\mathrm{PGI}_{2}$ pathway is also altered by $\mathrm{Xyl}-\mathrm{B}$, indomethacin (IMC, $10 \mu \mathrm{mol} / \mathrm{L}$ ) was used to inhibit $\mathrm{PGI}_{2}$ production by depressing COX-1, while methylene blue (MB, $10 \mu \mathrm{mol} / \mathrm{L})$ was applied to inhibit
sGC to interrupt the NO-sGC-cGMP pathway. As shown in Figure 3B, IMC did not alter the inhibitory effect of Xyl-B on vasocontractions provoked by Phe $(1 \mu \mathrm{mol} / \mathrm{L})$, which was significantly reversed by $\mathrm{MB}$, indicating that the NO-sGC-cGMP pathway mediates the anti-hypertensive effect of Xyl-B rather than $\mathrm{PGI}_{2}$.

In addition, we detected eNOS activity in HUVECs, which was measured as the conversion $\%$ of $\left[{ }^{3} \mathrm{H}\right] L$-citrulline transformed from $\left[{ }^{3} \mathrm{H}\right] \mathrm{L}$-arginine. As shown in Figure 4A, Xyl-B increased endothelial eNOS bioactivity in a dose- and timedependent manner. Moreover, Xyl-B increased the cGMP level of co-cultured VSMC-HUVECs, but not mono-cultured VSMCs (Figure 4B).

\section{Xyl-B attenuates smooth muscle cell (SMC) $\mathrm{Ca}^{2+}$ signaling}

As shown above, Xyl-B inhibited vascular constriction in response to $\mathrm{KCl}$ and Phe and this effect could not be completely eliminated by pre-incubation with L-NAME, suggesting that Xyl-B worked through other pathways or factors in addition to NO. As $\mathrm{PGI}_{2}$ production in the endothelium was excluded (Figure 5), the intracellular $\mathrm{Ca}^{2+}$ concentration $\left(\left[\mathrm{Ca}^{2+}\right]_{\mathrm{i}}\right)$ of smooth muscle cells, which plays a critical role during vasoconstriction, was speculated to be involved in this process. $\mathrm{KCl}$ and Phe elevate the $\left[\mathrm{Ca}^{2+}\right]_{\mathrm{i}}$ in different manners: $\mathrm{KCl}$ induces depolarization of SMCs, resulting in voltage-dependent $\mathrm{Ca}^{2+}$ channel activation, while Phe increases the $\left[\mathrm{Ca}^{2+}\right]_{i}$ by stimulating $\mathrm{Ca}^{2+}$ release from the ER and triggering store-operated $\mathrm{Ca}^{2+}$ entry. Our previous data indicated that some xyloketals, including xyloketal $\mathrm{B}$, can attenuate the L-type $\mathrm{Ca}^{2+}$ channel current in cultured newborn mouse hippocampal neurons ${ }^{[21]}$. Therefore, to investigate whether Xyl-B has an effect on the $\left[\mathrm{Ca}^{2+}\right]_{i}$ in SMCs, we first detected the effect of Xyl-B on $\mathrm{KCl}$-induced $\mathrm{Ca}^{2+}$ entry in SMCs and found that Xyl-B significantly suppressed the $\mathrm{Ca}^{2+}$ entry stimulated by $\mathrm{KCl}(60 \mathrm{mmol} / \mathrm{L})$, as shown in Figure 5A. Then, we determined the influence of Xyl-B on $\mathrm{Ca}^{2+}$ release from the ER using ryanodine $\left(10^{-7} \mathrm{~mol} / \mathrm{L}\right)$. As shown in Figure $5 \mathrm{~B}, \mathrm{Xyl}-\mathrm{B}$ pre-incubation obviously decreased ryanodineinduced vasoconstriction.

\section{Discussion}

This study demonstrates the antihypertensive effects of Xyl-B and the main findings are as follows: (1) $20 \mathrm{mg} \mathrm{kg} \mathrm{kg}^{-1}$ of Xyl-B significantly reduced SBP and DBP in the $2 \mathrm{~K} 2 \mathrm{C}$ hypertensive rat model, providing evidence for Xyl-B's in vivo antihypertensive effect for the first time. (2) Xyl-B decreased vascular tension in response to $\mathrm{KCl}$ and phenylephrine in thoracic aortas, which was partly reversed by incubation with L-NAME. (3) Xyl-B inhibited aortic vasocontractions through the endothelial NO-sGC-cGMP pathway rather than the $\mathrm{PGI}_{2}$ pathway. (4) Xyl-B regulated smooth muscle $\mathrm{Ca}^{2+}$ signaling by affecting both voltage-dependent $\mathrm{Ca}^{2+}$ entry (VDCC) and store-operated $\mathrm{Ca}^{2+}$ entry (SOCE) in vascular smooth muscle cells (Figure 6).

The $2 \mathrm{~K} 2 \mathrm{C}$ model is a widely used renovascular hypertension mode ${ }^{[12]}$ with significant value for assessing whether a drug has potential antihypertensive effects. Therefore, in this study, $2 \mathrm{~K} 2 \mathrm{C}$ hypertensive rats were used to evaluate Xyl-B's 

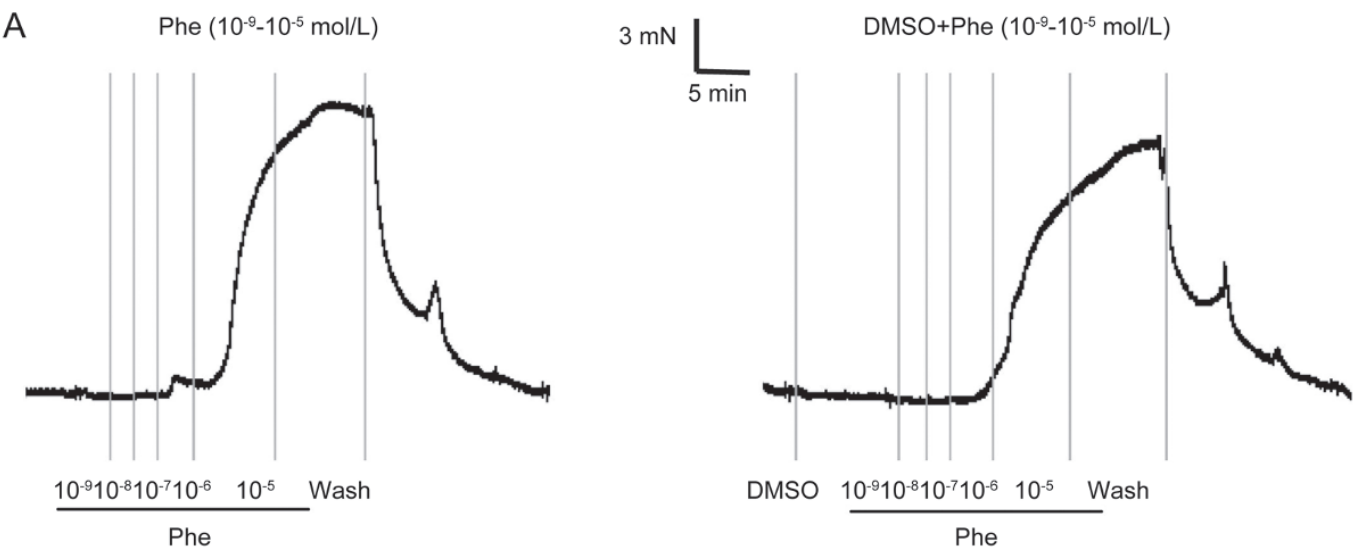

Xyl-B $(20 \mu \mathrm{mol} / \mathrm{L})+\mathrm{Phe}\left(10^{-9}-10^{-5} \mathrm{~mol} / \mathrm{L}\right)$

L-NAME + Xyl-B $(20 \mu \mathrm{mol} / \mathrm{L})+\mathrm{Phe}\left(10^{-9}-10^{-5} \mathrm{~mol} / \mathrm{L}\right)$
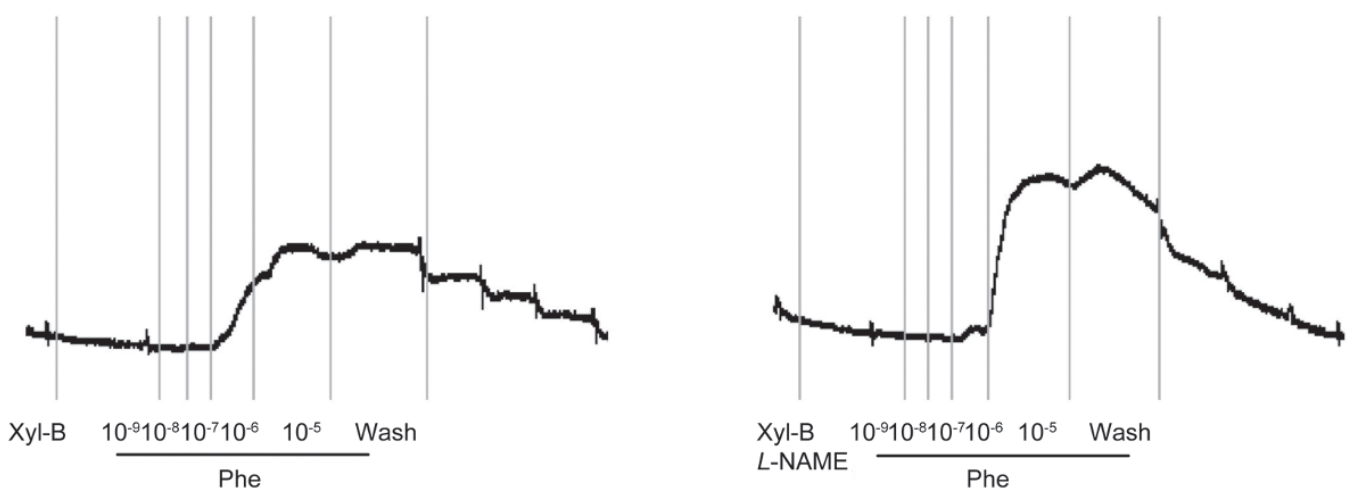

B

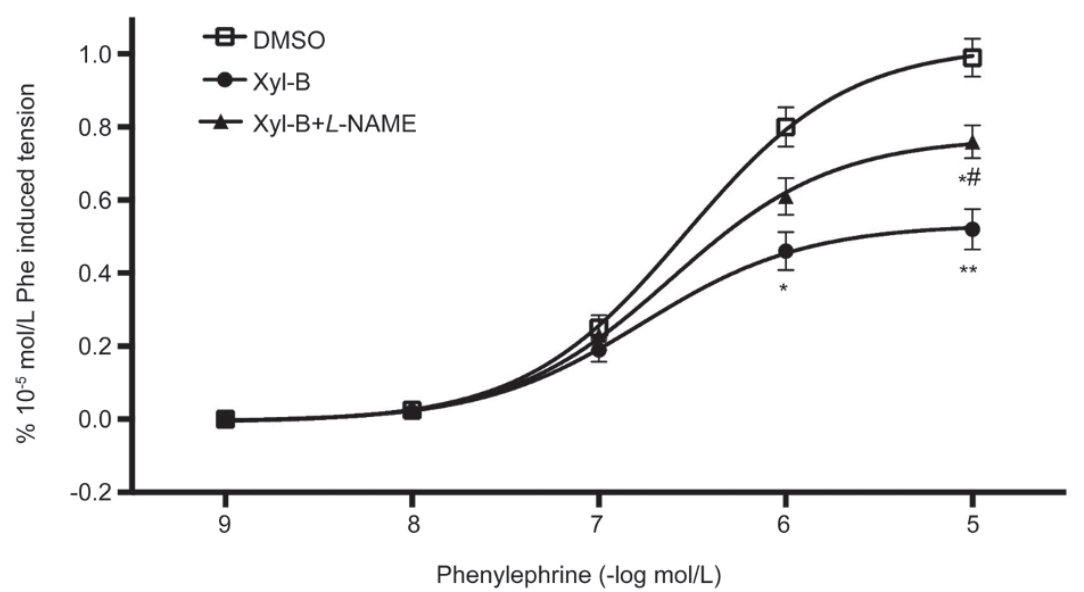

Figure 2. Effect of Xyl-B on phenylephrine (Phe) concentration-response curve for contraction in isolated rat thoracic aorta. Cumulative addition of Phe $\left(10^{-9}-10^{-5} \mathrm{~mol} / \mathrm{L}\right)$ induced a stepwise increase in aortic contraction. (A) Representative tension graph of Phe-induced aorta constriction with or without Xyl-B $(20 \mu \mathrm{mol} / \mathrm{L})$ pre-treatment $(10 \mathrm{~min})$ and the influence of L-NAME $(10 \mu \mathrm{mol} / \mathrm{L})$. Vascular tension caused by various concentrations $\left(10^{-9}, 10^{-8}, 10^{-7}\right.$, $10^{-6}, 10^{-5} \mathrm{~mol} / \mathrm{L}$ ) of Phe were recorded. (B) Statistical graph shows Xyl-B decreased Phe-induced tension at $10^{-6} \mathrm{~mol} / \mathrm{L}$ and $10^{-5} \mathrm{~mol} / \mathrm{L}$, and this reduction was partly reversed by L-NAME but not totally abolished. Data are expressed as mean \pm SEM. ${ }^{*} P<0.05,{ }^{* *} P<0.01$ vs DMSO group. ${ }^{\#} P<0.05$ vs $X y l-B$ group. $n=6$.

antihypertensive effects, and the results showed that Xyl-B could significantly decrease SBP and DBP in this model. Our previous studies demonstrated that Xyl-B can directly scavenge free radicals, promote endothelial $\mathrm{NO}$ release ${ }^{[7]}$ and protect the functions of mitochondria and the endothelium ${ }^{[6]}$. We suppose that these endothelium-dependent mechanisms may contribute to the antihypertensive effects of Xyl-B.

The endothelium regulates vascular homeostasis by modulating vasomotor tone through the production of several vasoactive mediators, including $\mathrm{NO}$ and prostacyclin ${ }^{[22,23]}$. In 
A

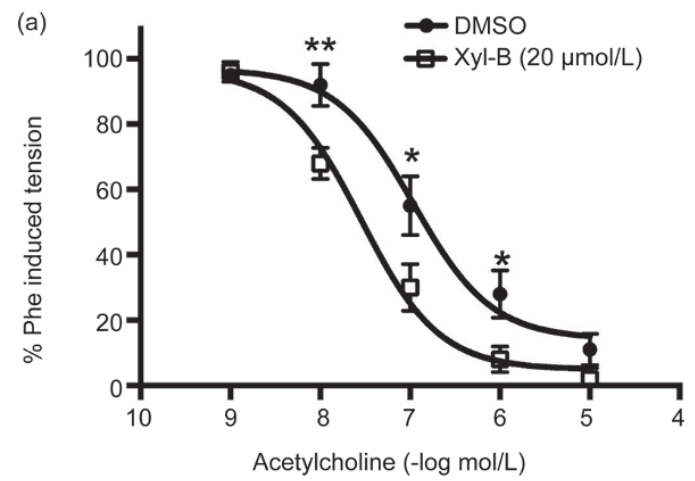

B (a)

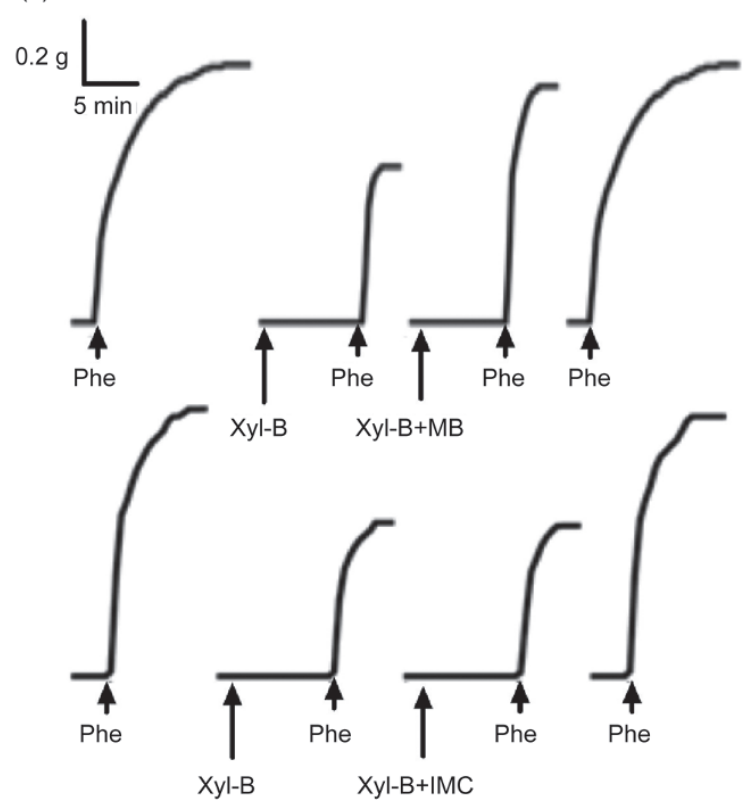

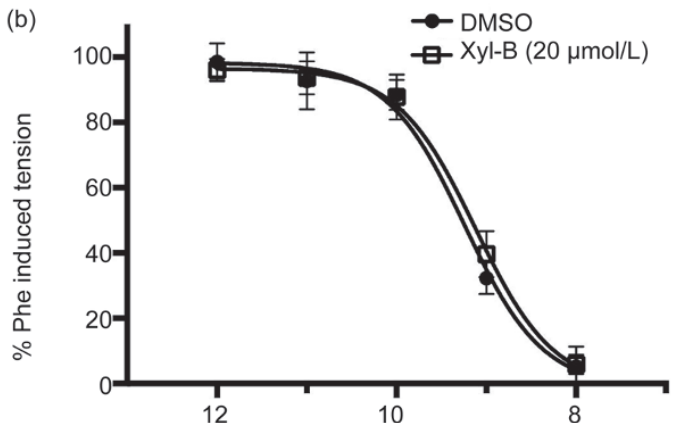

Sodium nitroprusside $(-\log \mathrm{mol} / \mathrm{L})$

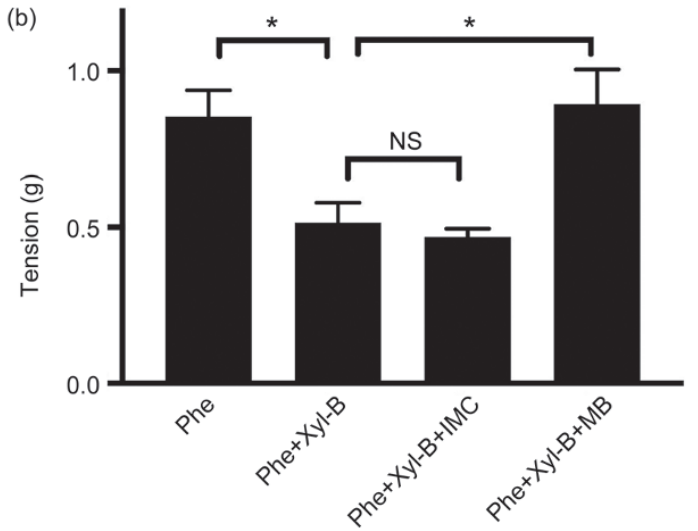

Figure 3. (A) Effect of Xyl-B on acetylcholine (ACh) or sodium nitroprusside (SNP) induced vaso-relaxation. Thoracic aorta rings were pre-contracted by Phe $(1 \mu \mathrm{mol} / \mathrm{L})$, then different concentration of (a) ACh $\left(10^{-9}-10^{-5} \mathrm{~mol} / \mathrm{L}\right)$ and $(\mathrm{b}) \mathrm{SNP}\left(10^{-12}-10^{-8} \mathrm{~mol} / \mathrm{L}\right)$ were cumulatively added. Data were expressed as mean \pm SEM in concentration-response curve. ${ }^{*} P<0.05,{ }^{* *} P<0.01$ vs DMSO group. $n=6$. (B) Xyl-B regulates vascular tone through endothelial NO-sGCcGMP pathway but not $\mathrm{PGI}_{2}$ pathway. (a) Original data show the influence of Indomethacin (IMC $10 \mu \mathrm{mol} / \mathrm{L}$ ) and methylene blue (MB, $10 \mu \mathrm{mol} / \mathrm{L}$ ) on the inhibition effect of Xyl-B on Phe-induced vasoconstriction. (b) Bar graph showed that IMC did not affect the inhibition effect of Xyl-B on vasocontraction provoked by Phe $(1 \mu \mathrm{mol} / \mathrm{L})$, which was reversed by MB. Data were expressed as mean \pm SEM. ${ }^{*} P<0.05$. $n=6$.

the development of hypertension, endothelial dysfunction precedes increases in blood pressure and predisposes patients to structural vascular changes ${ }^{[24,25]}$. Endothelial dysfunction is characterized by reduced NO levels or bioavailability ${ }^{[26]}$. Classically, $\mathrm{NO}$ is generated from $L$-arginine by the binding of $\mathrm{Ca}^{2+}$-calmodulin to eNOS ${ }^{[27]}$. NO released from endothelial cells stimulates soluble guanylate cyclase (sGC), leading to a sequential increase in the cGMP level in VSMCs ${ }^{[28]}$, which in turn activates cGMP-dependent protein kinase and leads to increased extrusion of $\mathrm{Ca}^{2+}$ from the cytosol in VSMCs and inhibition of the contractile machinery ${ }^{[28,29]}$. In the present study, both the eNOS inhibitor L-NAME and the sGC inhibitor MB suppressed the endothelium-dependent vasorelaxation induced by Xyl-B, but the cyclooxygenase blocker (indomethacin) could not. In addition, the cGMP-enhancing effect of Xyl-B was observed in VSMCs co-cultured with HUVECs, but not in the VSMC mono-culture. These data demonstrated that the endothelium-dependent vaso-relaxant activities of Xyl-B are attributed to the NO-sGC-cGMP signaling pathway rather than $\mathrm{PGI}_{2}$.

eNOS activity is known to be regulated by various intracellular signals, resulting in phosphorylation of eNOS ${ }^{[30]}$, and eNOS phosphorylation by the upstream PI3K/Akt pathway is required for efficient NO production ${ }^{[31,32]}$. Xyl-B has been shown to promote NO release and eNOS bioactivity in HUVECs ${ }^{[8]}$, which was confirmed in the present study through detection of the conversion ratio of $\left[{ }^{3} \mathrm{H}\right] \mathrm{L}$-arginine into $\left[{ }^{3} \mathrm{H}\right] L$-citrulline. Our recent study showed that Xyl-B could increase eNOS activity by regulating phosphorylation of Akt and eNOS at Ser ${ }^{177}$ and Thr ${ }^{495[10]}$. Although many aspects of Xyl-B as a novel drug candidate for protecting the vascular endothelium have been identified and found to be effective for 
A

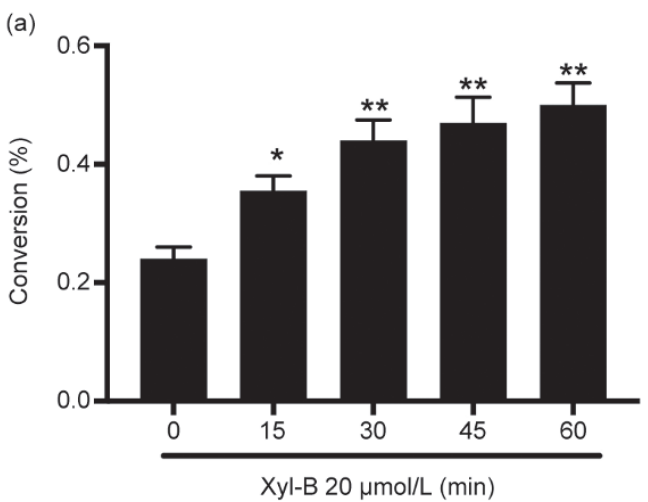

(b)

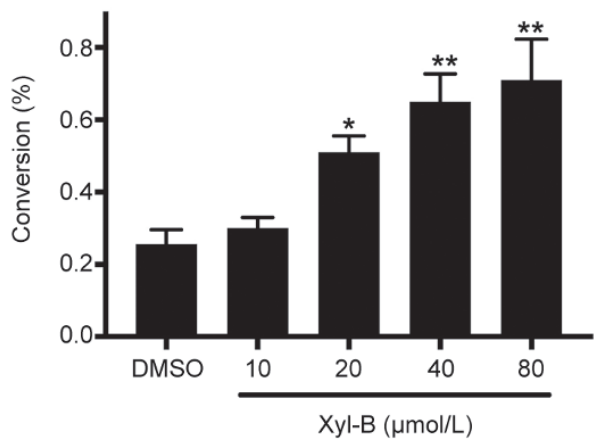

B

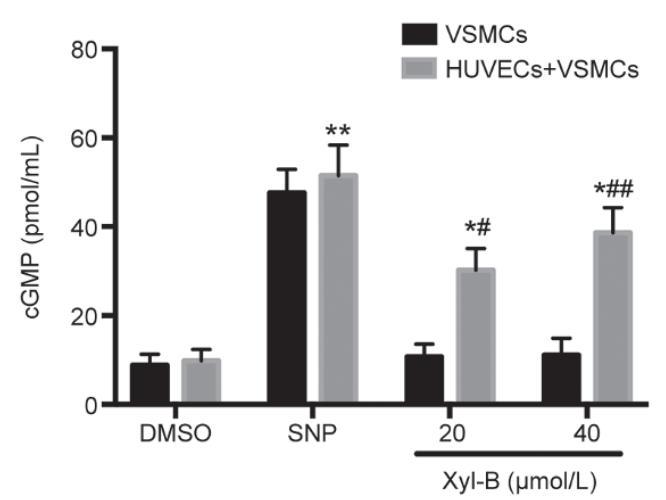

Figure 4. Effects of Xyl-B on eNOS activity in HUVECs and cGMP levels in VSMCs. (A) HUVECs were incubated in with $20 \mu \mathrm{mol} / \mathrm{L}$ of Xyl-B for 0 , 15, 30, 45 and $60 \mathrm{~min}$, or incubated for $30 \mathrm{~min}$ with Xyl-B of 0,10, 20, 40, $80 \mu \mathrm{mol} / \mathrm{L}$. Then eNOS activity was detected by measuring the formation of $\left[{ }^{3} \mathrm{H}\right] \mathrm{L}$-citrulline from $\left[{ }^{3} \mathrm{H}\right] \mathrm{L}$-arginine. eNOS activity was represented by conversion \%. (B) Vascular smooth muscle cells (VSMCs) were cultured alone or co-cultured with HUVECs. Both mono-cultured VSMCs and co-cultured VSMCs-HUVECs were incubated in the absence (DMSO) or presence of Xyl-B (20 and $40 \mu \mathrm{mol} / \mathrm{L})$ for $30 \mathrm{~min}$. SNP $(10 \mu \mathrm{mol} / \mathrm{L})$ served as positive control, then cGMP level was detected. Values are mean \pm SEM. ${ }^{*} P<0.05$, ${ }^{* *} P<0.01 \mathrm{vS}$ DMSO group. ${ }^{\#} P<0.05,{ }^{\# \#} P<0.01$ vs corresponding mono-cultured VSMCs. $n=6$.

the treatment of cardiovascular diseases such as atherosclero$\operatorname{sis}^{[7,8,10,11]}$, further experiments are required to confirm the role of the eNOS-dependent mechanism in the antihypertensive action of Xyl-B.

Our previous reports showed marked differences between endothelium-intact $\left(\mathrm{EC}_{50}=19 \mu \mathrm{mol} / \mathrm{L}\right)$ and endotheliumdenuded $\left(\mathrm{EC}_{50}=41 \mathrm{\mu mol} / \mathrm{L}\right)$ aortic rings after Xyl-B treatment $^{[10,11]}$, suggesting that Xyl-B may exert vaso-relaxant activities by endothelium-dependent mechanisms and by endothelium-independent mechanisms to a lesser extent. Interestingly, when we investigated the endothelial NO pathway using L-NAME, L-NAME could only partly reverse XylB's vaso-relaxant effect on Phe-stimulated pre-contracted aortic rings, but it could not eliminate this effect as a significant difference remained between the solvent control group and the Xyl-B+L-NAME group, indicating that other mechanisms independent of the NO system may be involved.

In addition to endothelial NO, vascular smooth muscle dysfunction is another important cause of hypertension ${ }^{[33]}$. Abnormal regulation of intracellular $\mathrm{Ca}^{2+}$ in VSMCs contributes to the pathogenesis of hypertension and also contributes to vascular and cardiac remodeling secondary to hyperten- sion. Two types of $\mathrm{Ca}^{2+}$ channels exist in VSMCs: VDCCs and voltage-independent $\mathrm{Ca}^{2+}$ channels (including receptoroperated $\mathrm{Ca}^{2+}$ channels and store-operated channels $)^{[34]} \cdot \mathrm{Ca}^{2+}$ channel blockers (CCBs) are clinically useful vasodilators and are widely used in the treatment of hypertension and related cardiovascular diseases ${ }^{[35]}$. CCBs lower blood pressure through a well-characterized mechanism of blocking L-type voltage-dependent $\mathrm{Ca}^{2+}$ channels in vascular cells to restore $\mathrm{Ca}^{2+}$ homeostasis ${ }^{[36]}$. Our previous data indicated that some xyloketals, including Xyl-B, can attenuate L-type $\mathrm{Ca}^{2+}$ currents in primary cultured hippocampal cells from newborn rats using patch-clamp experiments ${ }^{[21]}$, and the current study indicates that $\mathrm{Xyl}-\mathrm{B}$ dilated Phe- or $\mathrm{KCl}$-induced precontracted thoracic aortic rings. Phe-induced contractions in aortic rings are mainly mediated by $\mathrm{Ca}^{2+}$ entry via voltage-independent $\mathrm{Ca}^{2+}$ channels, such as SOCE, while $\mathrm{KCl}$ mainly activated the L-type $\mathrm{Ca}^{2+}$ channels (VDCCs). In the present study, we detected the effect of Xyl-B on $\mathrm{KCl}$-induced $\mathrm{Ca}^{2+}$ entry in SMCs and on ryanodine-induced aortic contraction. The results not only further confirmed the L-type $\mathrm{Ca}^{2+}$ channel-blocking effect of Xyl-B in smooth muscle cells but also indicated that Xyl-B may affect SOCE, warranting further investigation of the exact 

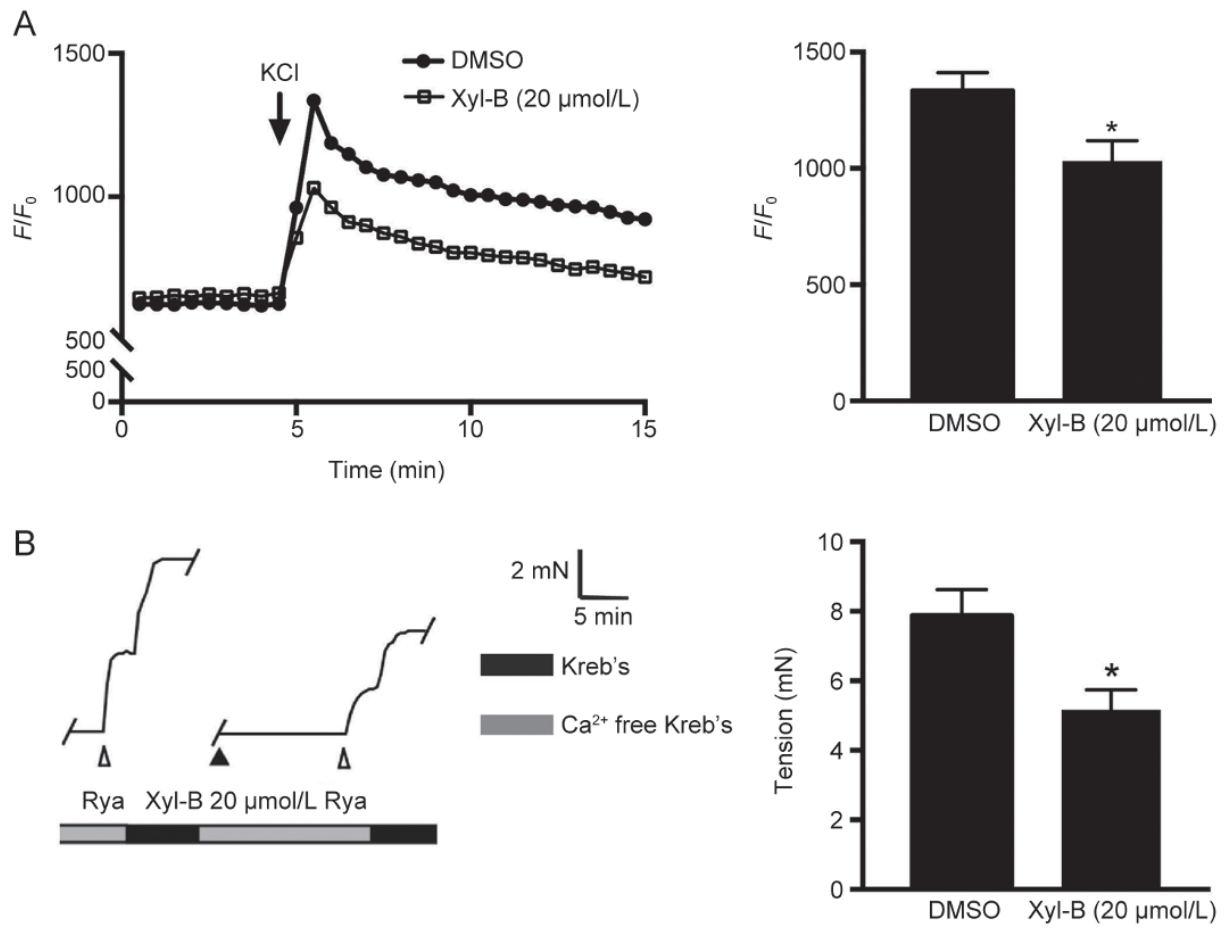

Figure 5. Xyl-B attenuates smooth muscle cells (SMCs) calcium signaling. (A) Xyl-B inhibits KCl-induced calcium entry in VSMCs. VSMCs were pretreated with Xyl-B $(20 \mu \mathrm{mol} / \mathrm{L})$ or DMSO for $10 \mathrm{~min}$ before stimulation with isotonic $60 \mathrm{mmol} / \mathrm{L} \mathrm{KCl}$. Realtime [Ca $\left.{ }^{2+}\right]_{\mathrm{i}}$ was determined using Fluo-3/AM staining and calculated every $30 \mathrm{~s}$ (left panel). Peak $\left[\mathrm{Ca}^{2+}\right]_{i}$ of both Xyl-B and DMSO treated cells were shown in bar graph. ${ }^{*} P<0.01$ vs DMSO group. Data were obtained from 6 independent experiments. (B) Aortic rings were placed in calcium-free solution with or without Xyl-B (20 $\mu$ mol/L), then ryanodine $\left(10^{-7} \mathrm{~mol} / \mathrm{L}\right)$ and calcium containing solution were added in order, aortic tension was recorded during this process. Bar graph shows that Xyl-B decreased ryanodine-induced vasoconstriction. Data are expressed as mean \pm SEM. Results were obtained from 6 independent experiments.

$\mathrm{Ca}^{2+}$ signaling mechanism. Additionally, new xyloketals such as xyloketal $\mathrm{F}$ and xyloketal $\mathrm{A}$ have shown strong inhibitory effects on VDCC in primary cultured rat hippocampal cells $(50.33 \% \text { and } 21.47 \% \text {, respectively })^{[21]}$, but they had no effects on $\mathrm{KCl}$-induced contractions of rat thoracic aortic rings and their effects could be completely inhibited by nifedipine (data not shown). Among the screened xyloketal compounds, only Xyl-B showed significant antihypertensive effects with minor inhibitory effects on VDCCs in newborn rat hippocampal cells $(12.05 \%)$. These data suggest that the influence of Xyl-B on $\mathrm{Ca}^{2+}$ signaling in different tissues and cell types should be interpreted with caution. Based on the current results, whether regulation of $\mathrm{Ca}^{2+}$ signaling by $\mathrm{Xyl}-\mathrm{B}$ is the predominant mechanism of its effects in hypertension remains unknown.

Furthermore, during the development of renovascular hypertension, major structural alterations in the heart include left ventricular (LV) hypertrophy and fibrosis ${ }^{[13]}$. The cardiovascular remodeling associated with hypertension involves oxidative stress and enhanced matrix metalloproteinase (MMP) expression/activity ${ }^{[13]}$. CCBs shows beneficial effects on left ventricular (LV) hypertrophy and fibrosis via antioxidative mechanisms ${ }^{[37]}$. The data observed in our current study show that Xyl-B can reverse cardiac remodeling and fibrosis in $2 \mathrm{~K} 2 \mathrm{C}$ rats (unpublished data). Therefore, Xyl-B not only attenuates the high $\mathrm{BP}$ level in hypertension but may also ameliorate the complications of hypertension.

In summary, this is the first study to demonstrate that Xyl-B can reduce blood pressure in $2 \mathrm{~K} 2 \mathrm{C}$ renovascular hypertensive rats. Our results suggest that Xyl-B induces relaxation in rat aortic rings through an endothelium-dependent pathway mediated by the NO-sGC-/cGMP pathway and through an endothelium-independent pathway involving VDCC blockade mediated by $\mathrm{Ca}^{2+}$ entry in VSMCs. One study limitation is that neither the NO-sGC-/cGMP pathway nor the $\mathrm{Ca}^{2+}$ signaling mechanisms underlying Xyl-B's antihypertensive effects were verified in VSMCs. Therefore, the mechanisms of the antihypertensive action of Xyl-B should be interpreted with caution, and further in vivo studies are warranted to determine the precise mechanism. Taken together, the findings of this study support Xyl-B as a potential new drug candidate for the treatment of hypertension.

\section{Acknowledgments}

This study was supported by grants from the National Natural Science Foundation of China (№ 81370897 and 81402926), the NSFC-CIHR China-Canada Joint Health Research Initiative Proposal (No 81361128011), the CIHR-NSFC China-Canada Joint Health Research Initiative (CIHR, FRN \#132571), the National Key New Drug Creation Program (No 2009ZX09103039), the Research Funds for Provincial Key Laboratory from the Department of Education of Guangdong Province (No 


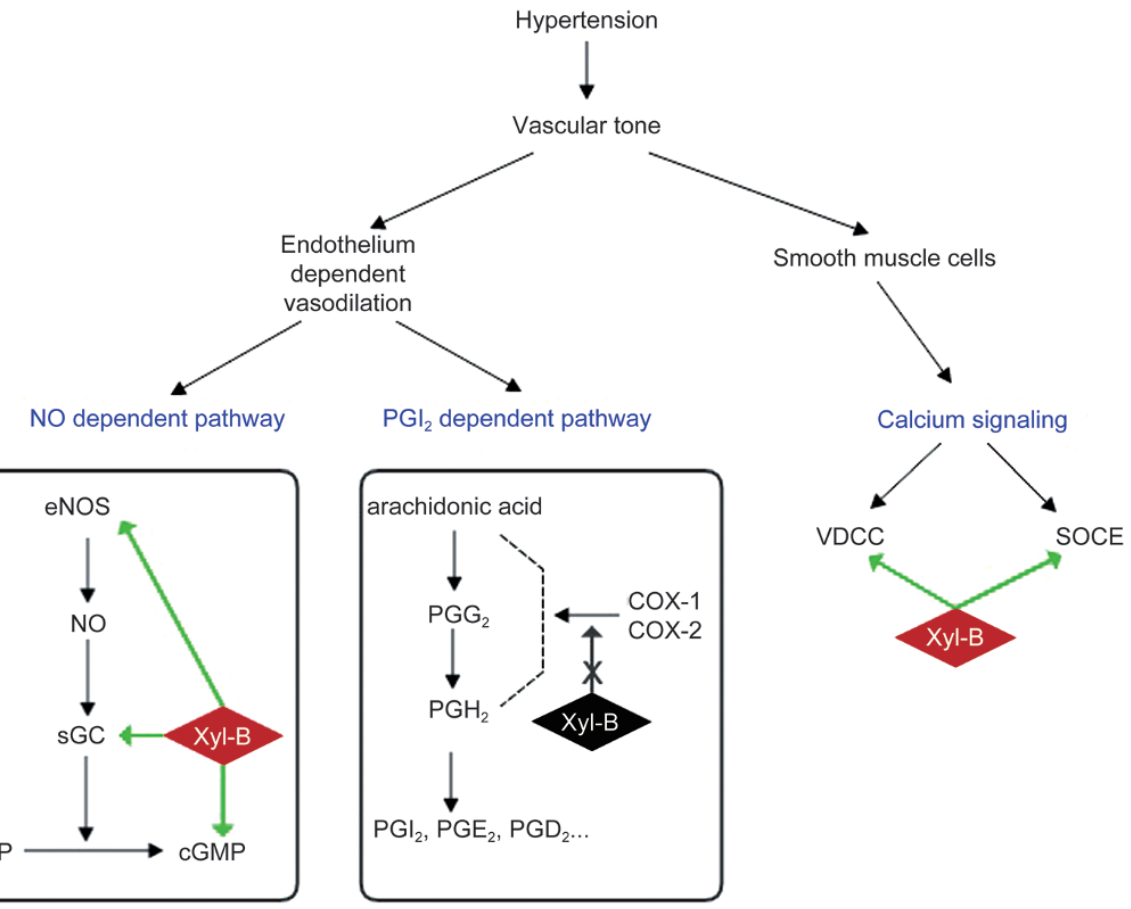

Figure 6. Summary of the vasoconstriction signal transduction pathways showing points of intervention by Xyl-B. Vascular tone is the result of joint regulation of endothelium and smooth muscle and endothelium-dependent vasodilation is regulated primarily by NO-sGC-cGMP signaling cascade and prostacyclin $\left(\mathrm{PGI}_{2}\right)$ in vascular smooth muscle cells. $\mathrm{PGI}_{2}$ is produced by the cyclooxygenases, COX-1 and cOX-2, which form the prostaglandin endoperoxide $\mathrm{PGG}_{2}, \mathrm{PGG}_{2}$ is converted to $\mathrm{PGH}_{2}$ which is then transformed enzymatically into $\mathrm{PGI}_{2}$ by prostacyclin synthase. Our present study demonstrated that Xyl-B affected eNOS activity and cGMP level, and SGC was also involved but $\mathrm{PGI}_{2}$ pathway was not. Additionally, as Xyl-B's inhibitory effect on vasoconstriction could not be completely explained by endothelial pathway, we furtherly found that Xyl-B regulated vascular tone by affecting both voltage dependent calcium entry (VDCC) and store-operated calcium entry (SOCE) in vascular smooth muscle cells.

50000-3211105), the Guangdong Natural Science Foundation (No 2016A030313293), Guangdong Provincial Department of Science and Technology (No 2016A050502023, No 2017A020215104) and the Science and Technology Program of Guangzhou (№ 201509010012).

\section{Author contribution}

Yao-min DU and Yong-cheng LIN conceived and designed the experiments; Li-yan ZHAO, Jie LI, Wei-feng MENG, Guo-hao WANG and Wen-liang CHEN performed the experiments; Li-yan ZHAO, Hong-shuo SUN and Xiong-qing HUANG analyzed the data; Xiao-fei LV and Ji-yan PANG contributed reagents and materials; Li-yan ZHAO and Guan-lei WANG wrote the paper.

\section{References}

1 Montezano AC, Dulak-Lis M, Tsiropoulou S, Harvey A, Briones AM, Touyz RM. Oxidative stress and human hypertension: vascular mechanisms, biomarkers, and novel therapies. Can J Cardiol 2015; 31: 631-41.

2 Guzik TJ, Touyz RM. Oxidative stress, inflammation, and vascular aging in hypertension. Hypertension 2017; 70: 660-7.

3 Schulz E, Jansen T, Wenzel P, Daiber A, Munzel T. Nitric oxide, tetrahydrobiopterin, oxidative stress, and endothelial dysfunction in hypertension. Antioxid Redox Signal 2008; 10: 1115-26.

4 Lin Y, Wu X, Feng S, Jiang G, Luo J, Zhou S, et al. Five unique compounds: xyloketals from mangrove fungus Xylaria sp. from the South China Sea coast. J Org Chem 2001; 66: 6252-6.

5 Pettigrew JD, Wilson PD. Synthesis of xyloketal A, B, C, D, and G analogues. J Org Chem 2006; 71: 1620-5.

6 Zhao J, Li L, Ling C, Li J, Pang JY, Lin YC, et al. Marine compound Xyloketal B protects PC12 cells against OGD-induced cell damage. Brain Res 2009; 1302: 240-7.

7 Chen WL, Qian Y, Meng WF, Pang JY, Lin YC, Guan YY, et al. A novel marine compound xyloketal $B$ protects against oxidized LDL-induced cell injury in vitro. Biochem Pharmacol 2009; 78: 941-50.

8 Li ZX, Chen JW, Yuan F, Huang YY, Zhao LY, Li J, et al. Xyloketal B exhibits its antioxidant activity through induction of HO-1 in vascular endothelial cells and zebrafish. Mar Drugs 2013; 11: 504-22.

9 Chen WL, Turlova E, Sun CL, Kim JS, Huang S, Zhong X, et al. Xyloketal B suppresses glioblastoma cell proliferation and migration in vitro through inhibiting TRPM7-regulated PI3K/Akt and MEK/ERK signaling pathways. Mar Drugs 2015; 13: 2505-25.

10 Zhao LY, Li J, Yuan F, Li M, Zhang Q, Huang YY, et al. Xyloketal B attenuates atherosclerotic plaque formation and endothelial dysfunction in apolipoprotein e deficient mice. Mar Drugs 2015; 13: 2306-26.

11 Xu Z, Li Y, Xiang Q, Pei Z, Liu X, Lu B, et al. Design and synthesis of novel xyloketal derivatives and their vasorelaxing activities in rat thoracic aorta and angiogenic activities in zebrafish angiogenesis screen. J Med Chem 2010; 53: 4642-53.

12 Zeng J, Zhang Y, Mo J, Su Z, Huang R. Two-kidney, two clip renovascular hypertensive rats can be used as stroke-prone rats. Stroke 1998; 29: 1708-13; discussion 13-4. 
13 Fang J, Xu SW, Wang P, Tang FT, Zhou SG, Gao J, et al. Tanshinone II-A attenuates cardiac fibrosis and modulates collagen metabolism in rats with renovascular hypertension. Phytomedicine 2010; 18: 58-64.

14 Kimura M, Sudhir K, Jones M, Simpson E, Jefferis AM, Chin-Dusting JP. Impaired acetylcholine-induced release of nitric oxide in the aorta of male aromatase-knockout mice: regulation of nitric oxide production by endogenous sex hormones in males. Circ Res 2003; 93: $1267-71$.

15 Casanello P, Sobrevia L. Intrauterine growth retardation is associated with reduced activity and expression of the cationic amino acid transport systems $y+/$ hCAT- 1 and $y+/$ hCAT-2B and lower activity of nitric oxide synthase in human umbilical vein endothelial cells. Circ Res 2002; 91: 127-34.

16 Xu S, Fu J, Chen J, Xiao P, Lan T, Le K, et al. Development of an optimized protocol for primary culture of smooth muscle cells from rat thoracic aortas. Cytotechnology 2009; 61: 65-72.

17 Li SJ, Sun NL. Regulation of intracellular $\mathrm{Ca}^{2+}$ and calcineurin by NO/ PKG in proliferation of vascular smooth muscle cells. Acta Pharmacol Sin 2005; 26: 323-8.

18 Shi XL, Wang GL, Zhang Z, Liu YJ, Chen JH, Zhou JG, et al. Alteration of volume-regulated chloride movement in rat cerebrovascular smooth muscle cells during hypertension. Hypertension 2007; 49: 1371-7.

19 Wang $M$, Yang $\mathrm{H}$, Zheng LY, Zhang Z, Tang YB, Wang GL, et al. Downregulation of TMEM16A calcium-activated chloride channel contributes to cerebrovascular remodeling during hypertension by promoting basilar smooth muscle cell proliferation. Circulation 2012; 125: $697-707$.

20 Nava E, Llorens S. The paracrine control of vascular motion. A historical perspective. Pharmacol Res 2016; 113: 125-45.

21 Wu XY, Liu XH, Lin YC, Luo JH, She ZG, Li HJ, et al. Xyloketal F: A strong L-calcium channel blocker from the mangrove fungus Xylaria $\mathrm{sp}$ (\#2508) from the South China Sea coast. Eur J Org Chem 2005; 19 : 4061-4.

22 Vane JR, Anggard EE, Botting RM. Regulatory functions of the vascular endothelium. N Engl J Med 1990; 323: 27-36.

23 Wei W, Chen ZW, Yang Q, Jin H, Furnary A, Yao XQ, et al. Vasorelaxation induced by vascular endothelial growth factor in the human internal mammary artery and radial artery. Vascul Pharmacol 2007; 46: 253-9.

24 Noll G, Wenzel RR, Schneider M, Oesch V, Binggeli C, Shaw S, et al. Increased activation of sympathetic nervous system and endothelin by mental stress in normotensive offspring of hypertensive parents. Circulation 1996; 93: 866-9.

25 Taddei S, Virdis A, Mattei P, Salvetti A. Vasodilation to acetylcholine in primary and secondary forms of human hypertension. Hypertension 1993; 21: 929-33.

26 Weil BR, Stauffer BL, Greiner JJ, DeSouza CA. Prehypertension is associated with impaired nitric oxide-mediated endotheliumdependent vasodilation in sedentary adults. Am J Hypertens 2011; 24: 976-81.

27 Fleming I, Bauersachs J, Busse R. Calcium-dependent and calciumindependent activation of the endothelial NO synthase. J Vasc Res 1997; 34: 165-74.

28 Sausbier M, Schubert R, Voigt V, Hirneiss C, Pfeifer A, Korth M, et al. Mechanisms of NO/cGMP-dependent vasorelaxation. Circ Res 2000; 87: 825-30.

29 Chang GJ, Lin TP, Ko YS, Lin MS. Endothelium-dependent and -independent vasorelaxation induced by $\mathrm{CIJ}-3-2 \mathrm{~F}$, a novel benzylfuroquinoline with antiarrhythmic action, in rat aorta. Life Sci 2010; 86: 869-79.

30 Rafikov R, Fonseca FV, Kumar S, Pardo D, Darragh C, Elms S, et al. eNOS activation and NO function: structural motifs responsible for the posttranslational control of endothelial nitric oxide synthase activity. J Endocrinol 2011; 210: 271-84.

31 Dimmeler S, Fleming I, Fisslthaler B, Hermann C, Busse R, Zeiher AM. Activation of nitric oxide synthase in endothelial cells by Aktdependent phosphorylation. Nature 1999; 399: 601-5.

32 Somani BL, Singh N, Singh VB. Study of Salmonella gallinarum infection in chicks by gas liquid chromatography. Indian J Exp Biol 1975; 13: 503-5.

33 Hixon ML, Gualberto A. Vascular smooth muscle polyploidization-from mitotic checkpoints to hypertension. Cell Cycle 2003; 2: 10510.

34 Guibert C, Ducret T, Savineau JP. Voltage-independent calcium influx in smooth muscle. Prog Biophys Mol Biol 2008; 98: 10-23.

35 Clunn GF, Sever PS, Hughes AD. Calcium channel regulation in vascular smooth muscle cells: synergistic effects of statins and calcium channel blockers. Int J Cardiol 2010; 139: 2-6.

36 Godfraind T, Miller R, Wibo M. Calcium antagonism and calcium entry blockade. Pharmacol Rev 1986; 38: 321-416.

37 Hasegawa H, Takano H, Kohro T, Ueda K, Niitsuma Y, Aburatani H, et al. Amelioration of hypertensive heart failure by amlodipine may occur via antioxidative effects. Hypertens Res 2006; 29: 719-29. 